\title{
Measurement Technique of Distribution of Power Generation Current Using Static Magnetic Field around Polymer Electrolyte Fuel Cell by 3D Inverse Problem FEM
}

\author{
Tomoaki Katou ${ }^{1, *}$, Yuji Gotoh ${ }^{1}$, Norio Takahashi ${ }^{2}$ and Masaaki Izumi ${ }^{3}$ \\ ${ }^{1}$ Department of Mechanical and Energy System Engineering, Faculty of Engineering, Oita University, Oita 870-1192, Japan \\ ${ }^{2}$ Department of Electrical and Electronic Engineering, Graduate School of Natural Science \& Technology, \\ Okayama University, Okayama 700-8530, Japan \\ ${ }^{3}$ Department of Mechanical System Engineering, Faculty of Environmental Engineering, \\ The University of Kitakyushu, Kitakyushu 808-0135, Japan
}

A polymer electrolyte fuel cell (PEFC) is a practical clean energy converter. In order to improve power generation state in PEFC is needed. The power generation performance of PEFC depends on mass transfer in it. Thus, it is important to clarify the current distribution that has close relation to the mass transfer. Especially, the measurement of the power generation current in MEA (membrane electrode assembly) inside the PEFC is needed. The power generation current generates the static magnetic field around the PEFC. Therefore, the distribution of power generation current inside MEA can be determined using the static magnetic field around the fuel cell.

In this paper, non-contacting technique for distribution of power generation current inside the MEA using the distribution of the static magnetic field around the PEFC is examined. The proposed heuristic search method using 3D FEM (finite element method) is used for searching the current distribution inside the MEA. In the proposed heuristic search magnetic field analyzing method, the "ON" (current flow) or "OFF" (no current) of the generation current region only in MEA which consisted of 25 elements in the design variable. The distribution of power generation current in MEA is calculated from the proposed heuristic search analyzing method using the measured values of static magnetic field around the PEFC. By measuring the very small quantity space magnetic field distributed around the PEFC the distribution of power generation current in MEA with the fuel cell can be calculated using the proposed heuristic search method. [doi:10.2320/matertrans.I-M2011842]

(Received November 10, 2009; Accepted October 29, 2011; Published January 25, 2012)

Keywords: heuristic search, finite element method, current measurement, polymer electrolyte fuel cell, membrane electrode assembly

\section{Introduction}

A polymer electrolyte fuel cell (PEFC) with a quick power generation is going to be used for the power supply of an electric vehicle etc. The improvement of efficiency and durability are strongly required for the spread of the PEFC. The power generation performance of PEFC depends on the mass transfer of hydrogen, oxygen and steam in it. Thus, it is important to clarify the current distribution that has close relation to the mass transfer. Especially, the measurement of the power generation current in MEA (membrane electrode assembly) inside PEFC is necessary. The generation current distribution was measured by taking out currents through divided elements of MEA or a separator and externally measuring the current. ${ }^{1,2}$ )

On the other hand, the power generation current generates the static magnetic field around the fuel cell. Therefore, there is a possibility to determine the distribution of power generation current inside MEA using the static magnetic field around fuel cell. Some approaches to determine the current distribution using the outside field are reported. ${ }^{3,4)}$

In this paper, the non-contacting detection technique of distribution of power generation current inside MEA using the static magnetic field around the fuel cell is examined. The static magnetic field around the fuel cell can be measured using the magnetic sensor etc. In this research, the calculated static magnetic field by the forward 3D FEM analysis is used as the measured value in the proposed 3D heuristic search,

*Graduate Student, Oita University

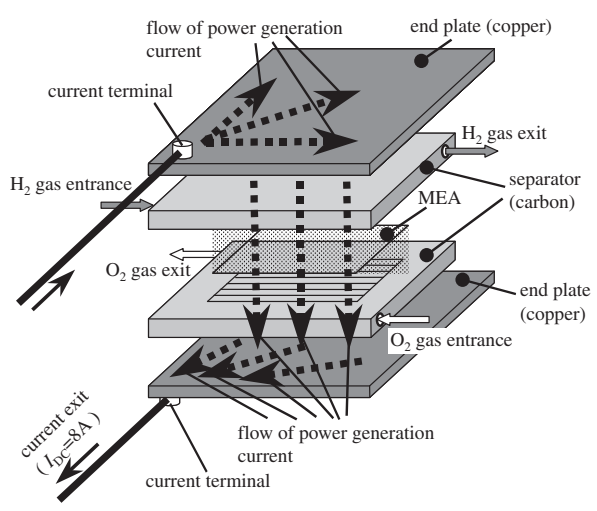

Fig. 1 Composition of polymer electrolyte fuel cell.

and the current distribution in MEA is determined. Moreover, the experimental verification is also carried out by using imitation fuel cell.

\section{Model and Method of Analysis}

\subsection{Model of PEFC and measurement domain of flux density}

Figure 1 shows the structure of the PEFC. This composed of a pair of end plate (copper plate), a pair of separator (carbon) that is the passages of hydrogen and oxygen, and a sheet of MEA. The fuel cell was arranged horizontally. Figure 2 shows the analyzed model of the fuel cell using the 3D FEM. The MEA is a sheet and its size is $50 \mathrm{~mm} \times$ 


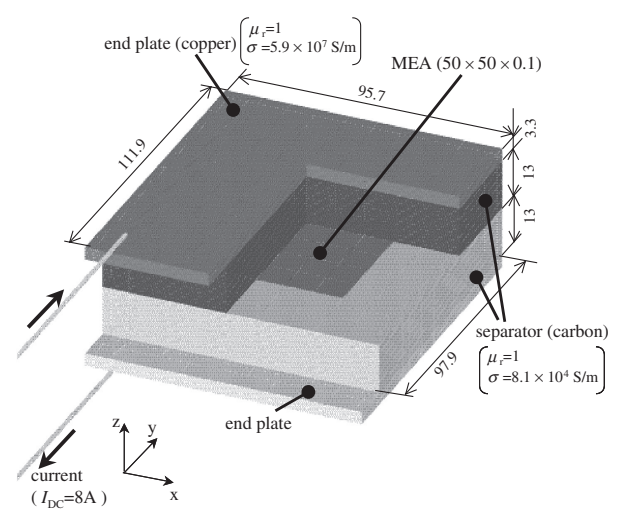

Fig. 2 Model of PEFC (unit: mm).

Table 1 Condition of forward analysis.

\begin{tabular}{|c|c|}
\hline total generation current & $I_{\mathrm{dc}}=8 \mathrm{~A}$ \\
\hline end plate (copper) & $\begin{array}{l}\text { width in the } x \text {-direction }=95.7 \mathrm{~mm} \\
\text { length in the } y \text {-direction }=111.9 \mathrm{~mm} \\
\text { thickness in the } z \text {-direction }=3.3 \mathrm{~mm} \\
\text { conductivity } \sigma=5.9 \times 10^{7} \mathrm{~S} / \mathrm{m}\end{array}$ \\
\hline separator (carbon) & $\begin{array}{l}\text { width in the } x \text {-direction }=95.7 \mathrm{~mm} \\
\text { length in the } y \text {-direction }=95.7 \mathrm{~mm} \\
\text { thickness in the } z \text {-direction }=13 \mathrm{~mm} \\
\text { conductivity } \sigma=8.1 \times 10^{4} \mathrm{~S} / \mathrm{m}\end{array}$ \\
\hline MEA (copper) & $50 \mathrm{~mm} \times 50 \mathrm{~mm} \times 0.1 \mathrm{~mm}(25$ elements $)$ \\
\hline nodes and elements & 29952,26979 \\
\hline Convergence criterion & $\begin{array}{l}\text { Incomplete Cholesky Conjugate Gradient } \\
\text { Method (ICCG method): } 1.0 \times 10^{-4} \\
\text { Newton-Raphson Method: } 1.0 \times 10^{-4}\end{array}$ \\
\hline
\end{tabular}

(a)

(b)

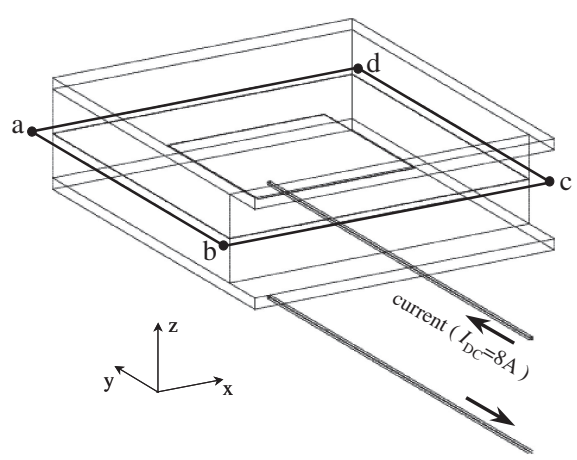

Fig. 3 Position of magnetic measurements.

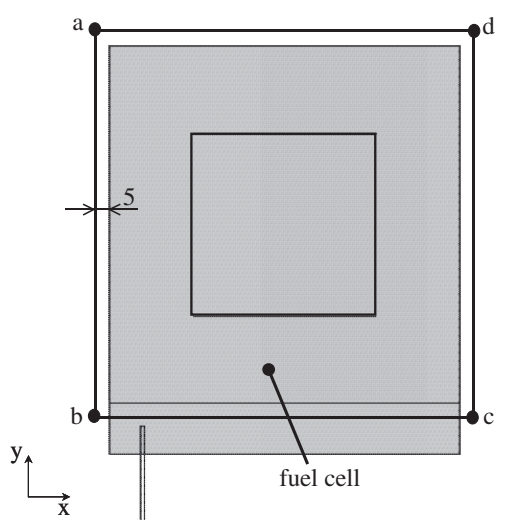

(a) Bird's view (b) $x, y$-plane (unit: mm).

$50 \mathrm{~mm} \times 0.1 \mathrm{~mm}$. The conductivities of the end plate and separator are $5.9 \times 10^{7} \mathrm{~S} / \mathrm{m}$ and $8.1 \times 10^{4} \mathrm{~S} / \mathrm{m}$, respectively. The total amount of power generated by this fuel cell is $\mathrm{dc}$ $8 \mathrm{~A}$. The number of elements in MEA is 25 . Table 1 shows the condition of analysis.

The flux density $B$ in or around the fuel cell is produced by power generation current inside the fuel cell. Therefore, there is a possibility that the distribution of power generation current inside the fuel cell or the MEA can be determined using $B$ around the fuel cell. The static $B_{x}, B_{y}$ and $B_{z}$ along the line a-b-c-d shown in Fig. 3 are measured. The $x$-, $y$-, and $z$-components of static magnetic field calculated in the forward analysis are used in 3D heuristic search. ${ }^{5}$ )

\subsection{Proposed heuristic search}

There are various techniques in the heuristic search method. When the Rosenbrock method ${ }^{6)}$ is used for this model, solutions different from a correct answer were obtained. Moreover, even when the Evolution Strategy ${ }^{7)}$ using Tikhonov's method is used, the optimal solution was not obtained if number of design variables is large.

Then, a heuristic search which determines an "ON" or "OFF" region of the generation current in MEA which satisfies the specified flux distribution is introduced. The iteration process of calculation is shown in Figs. 4(a) to 4(c) and the algorithm is as follows:

(a) process 1

An "OFF" (no current) element of black or gray color domain in shown Fig. 4 is generated in MEA, and the following objective function $W$ is calculated:

$$
\begin{array}{r}
W^{k}=\sum_{i=1}^{n}\left\{\left(B_{i x}-B_{0 x}\right)^{2}+\left(B_{i y}-B_{0 y}\right)^{2}+\left(B_{i z}-B_{0 z}\right)^{2}\right\} \\
(k=1,2,3, \ldots)
\end{array}
$$

where $n$ is the number of elements in the target domain around the fuel cell shown in Fig. 3. $B_{i x}, B_{i y}$ and $B_{i z}$ are the $x$-, $y$-, and $z$-components of the flux density calculated using 3D FEM. " $k$ " of the superscript of $W$ denotes the calculation process $k . B_{0 x}, B_{0 y}$ and $B_{0 z}$ are the $x$-, $y$-, and $z$-components of flux density calculated by forward analysis (corresponding to a measured value). Twenty-five kinds of calculations of $W$ are carried out for each "OFF" (25 elements) in MEA. The smallest five $W_{\mathrm{s}}$ are chosen from all $W^{1}$. These five $W^{1} \mathrm{~s}$ are defined as $W^{1}$ No.1 to $W^{1}$ No.5. Figure 4(a) shows the example of distribution of ON/OFF

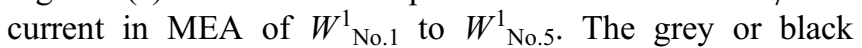
element denotes an "OFF" element. 
(a)

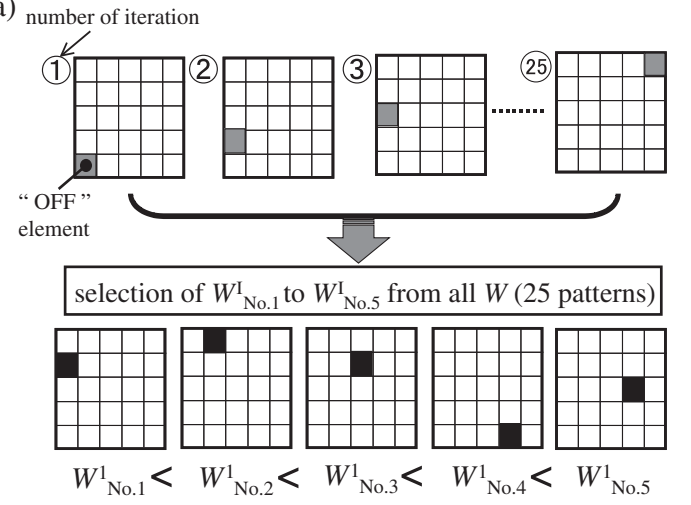

Process 1

(b)

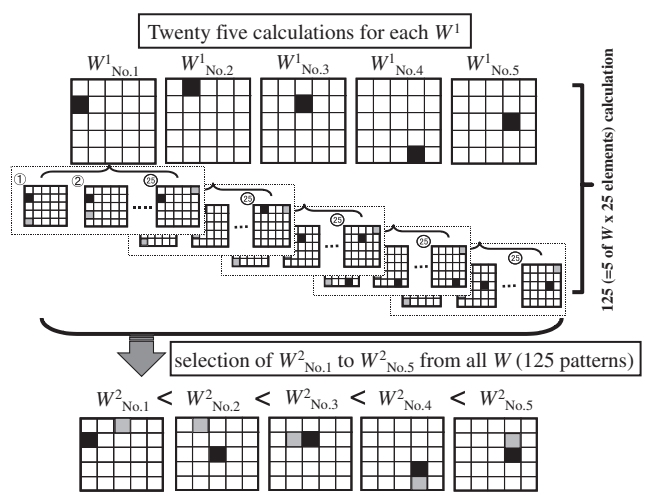

Process 2

(c)

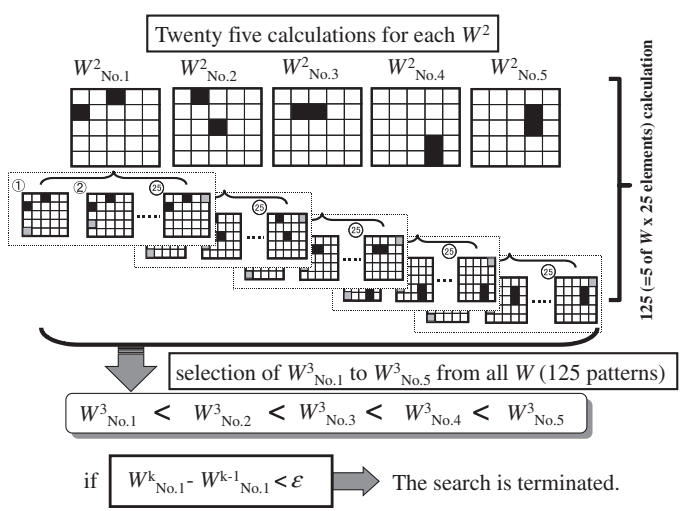

Process 3

Fig. 4 Iteration process using proposed heuristic search method.

(b) process 2

Twenty-five calculations are carried out by generating additional one "OFF" element for each $W^{1}$ as shown in Fig. 4(b). Then, new current distributions of $W^{2}{ }_{\text {No.1 } 1}$ to $W^{2}{ }_{\text {No. }} 5$ are searched among $5 \times 25=125$ patterns.

(c) process 3

The calculation of process 2 is iterated as shown in Fig. 4(c), and new current distributions of $W^{k}$ No.1 to $W^{k}$ No.5 at the process $k$ are obtained.

If $W^{k}$ No.1 to $W^{k-1}$ No.1 is less than $\varepsilon$ (small number, $\varepsilon$ is chosen as $\left.1.0 \times 10^{-20}\right)$, the search is terminated. (a)

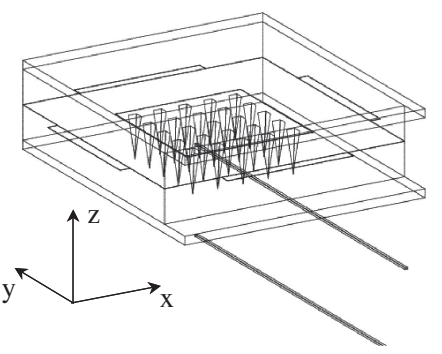

(b)

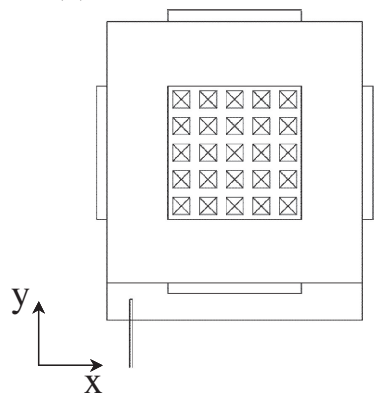

Fig. 5 Distribution of uniform power generation current in MEA. (a) Power generation current density in MEA (b) $x, y$-plane (each $\left.I_{\mathrm{dc}}=0.32 \mathrm{~A}\right)$.

(a)

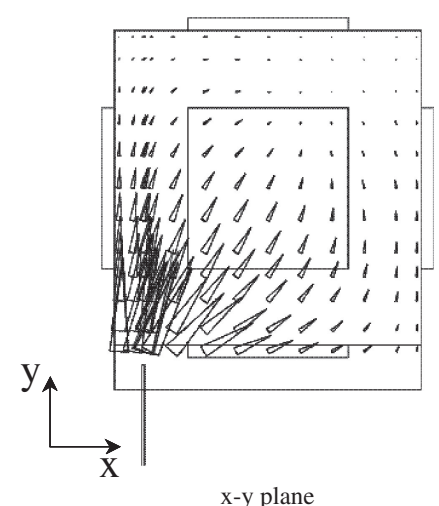

(b)

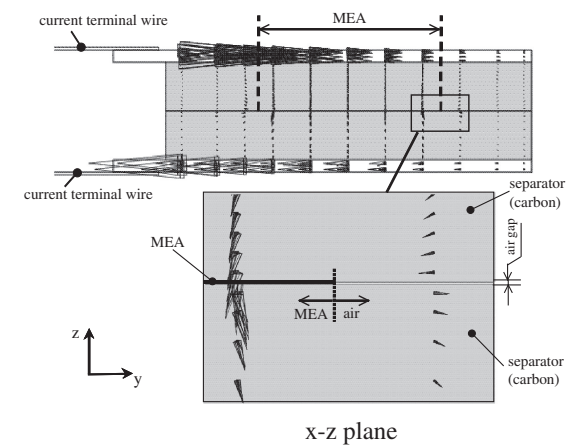

Fig. 6 Whole distribution of power generation current in the fuel cell.

\section{Result and Discussion of Heuristic Search}

\subsection{Forward analysis}

The distributions of current and static magnetic field at an ideal power generation (the current is generated in whole MEA) are calculated by the forward analysis. Figure 5 shows the distribution of uniform power generation current in MEA. The volume and direction of the current are shown by the volume and direction of the arrow sign in the figure. MEA is subdivided into 25 elements and the current of each element is $I_{\mathrm{dc}}=0.32 \mathrm{~A}(=8 \mathrm{~A} / 25$ elements $)$. Figure 6 shows the whole distribution of power generation current in the fuel cell. The volume and direction of the current are shown by 
(a)

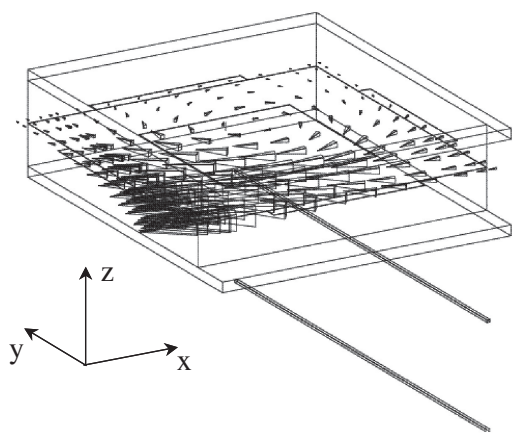

(b)

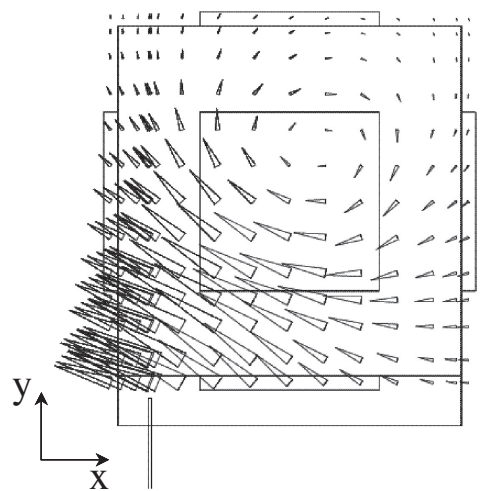

Fig. 7 Distribution of flux density around the fuel cell at ideal power generation. (a) Flux density only around MEA (b) $x-y$ plane $\left(|B|_{\max }=1.264 \times 10^{-4} \mathrm{~T}\right.$ and $|B|_{\min }=0.443 \times 10^{-4} \mathrm{~T}$ in domain of measurements $)$.

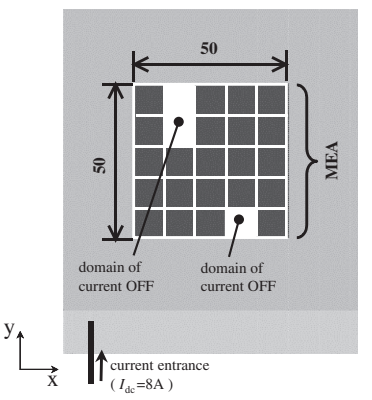

Fig. 8 Current distribution set up in forward analysis in MEA.

the volume and direction of the arrow sign in the figure. The current generated within MEA flows into a separator, an end plate, and a current terminal wire. As the conductivity of the end plate (copper) is larger than of the separator (carbon), the current density in the end plate is largest within the fuel cell. Figure 7 shows the distribution of flux density around MEA. The volume and direction of the flux density are shown by the volume and direction of the arrow sign in the figure. Since $|B|_{\max }$ and $|B|_{\min }$ in the measurement domain of flux density are $1.264 \times 10^{-4} \mathrm{~T}$ and $0.443 \times 10^{-4} \mathrm{~T}$, respectively, the quantity of flux density can be measured by Hall elements.

\subsection{Heuristic search}

In order to check the performance of the proposed heuristic method, the case when the currents of three elements in MEA are "OFF" as shown in Fig. 8 is examined. Figure 9 shows the distribution of power generation current in MEA calculated by the forward analysis. The volume and direction of the current are shown by the volume and direction of the arrow sign in the figure. The total current in MEA is $8 \mathrm{~A}$, and the current of each element is about $0.36 \mathrm{~A}(=8 \mathrm{~A} /$ 22 elements). Figure 10 shows the distribution of flux density around the fuel cell near MEA. The volume and direction of the flux density are shown by the volume and direction of the arrow sign in the figure. $|B|_{\max }$ and $|B|_{\min }$ in the measurement domain of flux density are $1.265 \times 10^{-4} \mathrm{~T}$ and $0.062 \times 10^{-4} \mathrm{~T}$, respectively.

Figure 11 shows the change of objective function $W$ at each iteration. The reducing histories of five kinds of

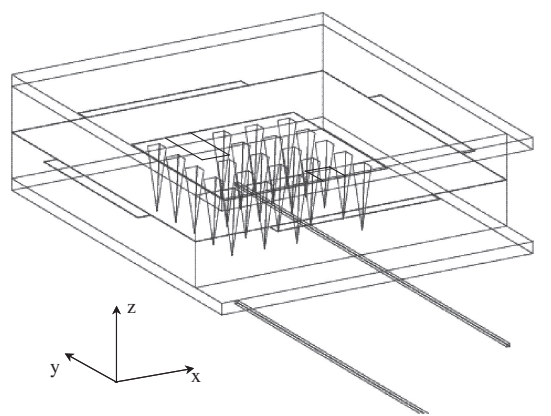

Fig. 9 Distribution of current density by forward FEM $\left(I_{\mathrm{dc}}=0.32 \mathrm{~A}\right)$.

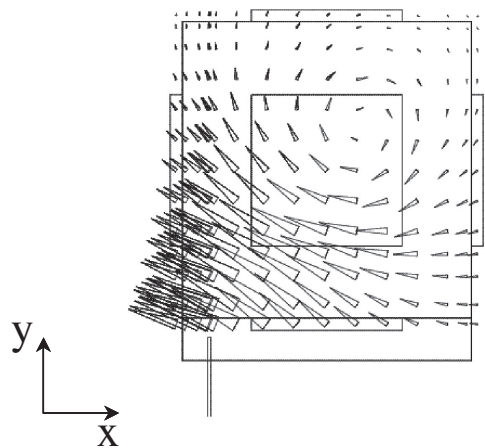

Fig. 10 Distribution of flux density around the fuel cell $\left(|B|_{\max }=\right.$ $1.264 \times 10^{-4} \mathrm{~T}$ and $|B|_{\min }=0.443 \times 10^{-4} \mathrm{~T}$ in domain of measurements).

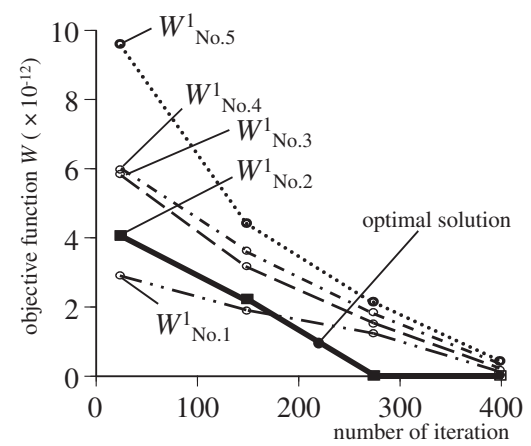

Fig. 11 Calculation process of an objective function $W$. 
(a)
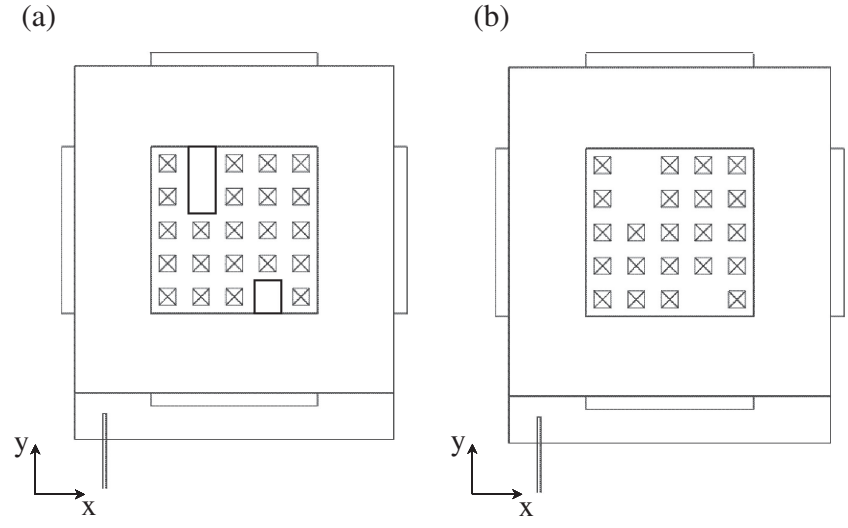

Fig. 12 Distribution of current destiny in MEA. (a) Forward analysis (b) proposed heuristic search.

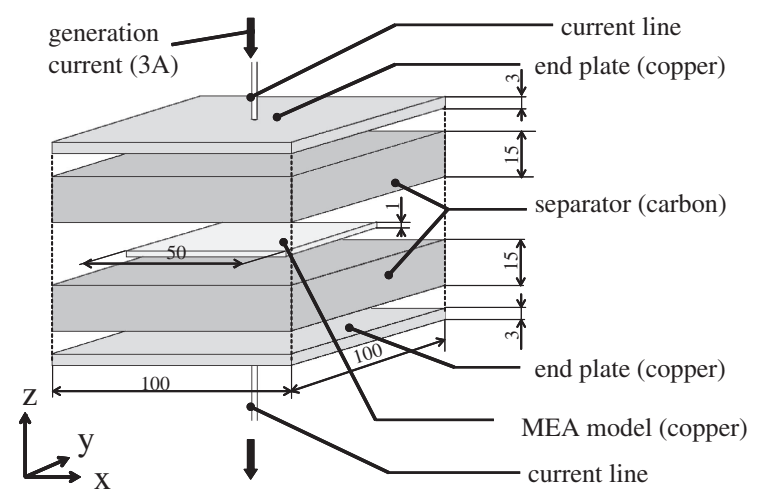

Fig. 13 Model of imitation PEFC for verification experiment (unit: mm).

objective functions $W^{1}$ No.1 to $W^{1}$ No.5, which were selected at the process 1, are monitored. The figure shows that the heuristic search is converged by about 400 iterations.

Figure 12 shows the comparison between the current distributions in MEA by the forward analysis and by the heuristic search. The volume and direction of the current are shown by the volume and direction of the arrow sign in the figure. The figure shows that the current distribution in MEA of the fuel cell can be obtained by the proposed heuristic search using the flux distribution around the fuel cell.

\section{Verification Experiment}

In this research, the imitation problem electrolyte fuel cell was created and the current distribution in the MEA using a copper plate was estimated. In the verification experiment, the "ON" (current flow) or "OFF" (no current) of the generation current region only in MEA which consisted of 25 elements. The verification experiment is carried out by using proposed heuristic search with the static magnetic field measured around the fuel cell.

\subsection{Model of imitation equipment of the fuel cell}

Figure 13 shows the imitation model of polymer electrolyte fuel cell for verification experiment. The structure of the model is a pair of end plate (copper plate), a pair of separator (carbon), a pair of current line and a sheet of MEA. In addition, the MEA in the fuel cell model was assumed by a

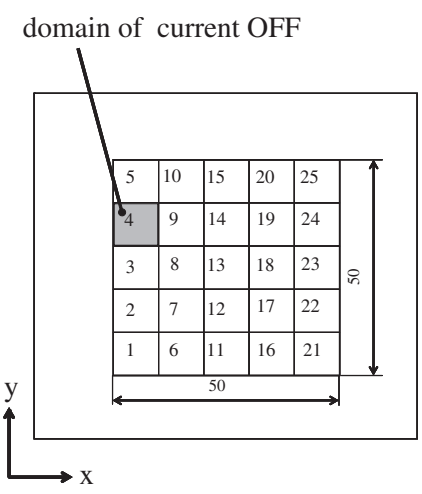

Fig. 14 One element of the current "OFF" in MEA model which consisted of 25 elements.

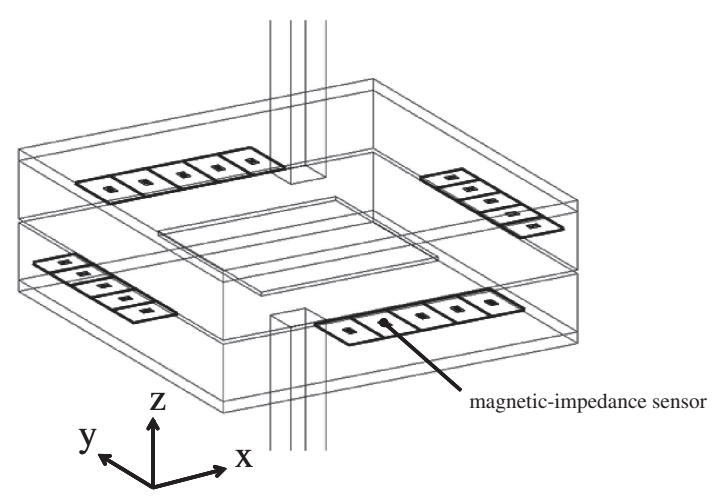

Fig. 15 Measurement model of static field density $B_{x}$ and $B_{y}$ of 20 positions around the fuel cell.

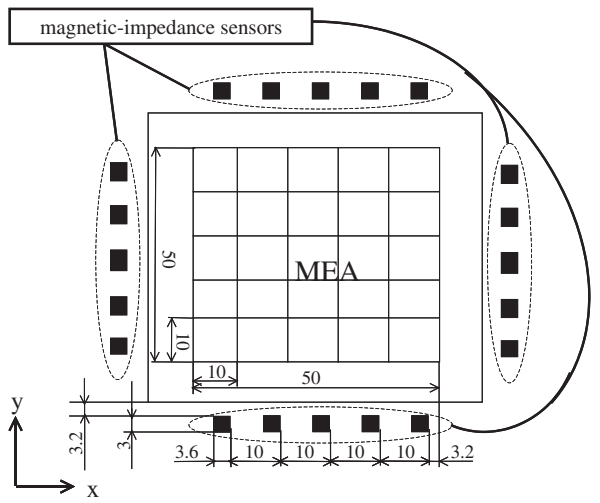

Fig. 16 Size and positions of 20 magnetic sensors.

copper plate in this experiment. The conductivities of the end plate, the separator and a sheet of MEA are $5.15 \times 10^{7} \mathrm{~S} / \mathrm{m}$, $6.26 \times 10^{4} \mathrm{~S} / \mathrm{m}$ and $4.6 \times 10^{7} \mathrm{~S} / \mathrm{m}$, respectively. In this imitation fuel cell, the direct-current $(3 \mathrm{~A})$ is flowed from upper side current line to a pair of end plate, a pair of separator and MEA model of copper. Figure 14 shows one element of the current "OFF" in MEA is which consisted of 25 elements. The static flux density $B_{x}$ and $B_{y}$ of 20 positions around the fuel cell shown in Fig. 15 are measured. Figure 16 shows the size and positions of 20 magnetic sensors around the MEA. The $x$ - and $y$-components of the static magnetic field by measurement are used in 3D heuristic search. An "OFF" (no current) element is generated in MEA, and the following objective function $W$ is calculated. 


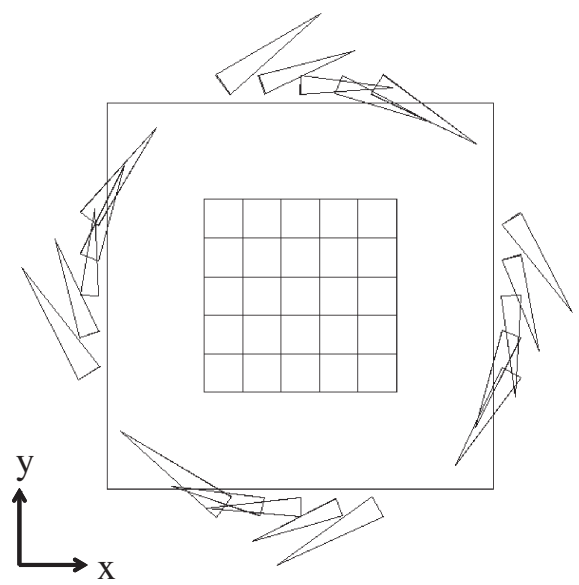

Fig. 17 Measurement distribution of static magnetic field around the fuel cell.

$$
\begin{array}{r}
W^{k}=\sum_{i=1}^{n}\left\{\left(B_{i x}-B_{0 x}\right)^{2}+\left(B_{i y}-B_{0 y}\right)^{2}\right\} \\
(k=1,2,3, \ldots)
\end{array}
$$

Where $n=20$ in the number of elements in the target domain around the fuel cell in Fig. 16. $\mathrm{B}_{0 x}$ and $B_{0 y}$ are $x$ - and $y$ components of the flux density measured by 20 magnetic sensors. $B_{i x}, B_{i y}$ and $k$ are similar to the formula (1).

\subsection{Result and discussion of verification experiment}

Figure 17 shows distribution of measurement results of the static magnetic field around the fuel cell. The volume and direction of the flux density are shown by the volume and direction of the arrow sign in the figure. The figure denotes that $|B|_{\max }$ and $|B|_{\min }$ in these distributions of the flux density are $9.3 \times 10^{-6} \mathrm{~T}$ and $7 \times 10^{-7} \mathrm{~T}$, respectively. These measured values are used for 3D heuristic search. Figure 18 shows current distribution in MEA is detected by $3 \mathrm{D}$ heuristic search FEM. The volume and direction of the current are shown by the volume and direction of the arrow sign in the figure. The figure denotes that $I_{\max }$ and $I_{\min }$ in these distributions of the current density are $16 \times 10^{2} \mathrm{~A} / \mathrm{m}^{2}$ and $4 \times 10^{2} \mathrm{~A} / \mathrm{m}^{2}$, respectively. The correct "OFF" region of No. 4 element is determined by proposed heuristic search. Figure 18 shows the calculation result of current distribution in the MEA using 3D heuristic search. Figure 18(b) denote that the calculated current distribution is in agreement with the domain of the current "OFF" in shown Fig. 14. This figure shows that the detection of the current distribution in MEA is possible from the distribution of static magnetic field around the fuel cell using the proposed 3D heuristic search. (a)

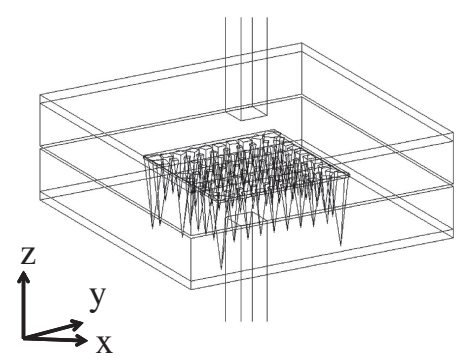

(b)

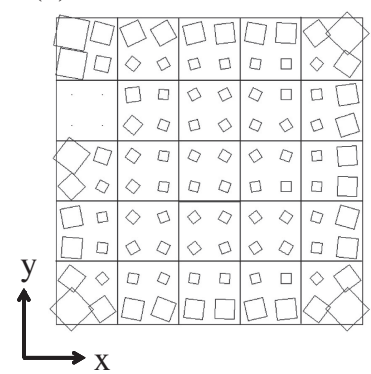

Fig. 18 Calculated result of current distribution in MEA by 3D heuristic search $\left(I_{\max }=16 \times 10^{2} \mathrm{~A} / \mathrm{m}^{2}, I_{\min }=4 \times 10^{2} \mathrm{~A} / \mathrm{m}^{2}\right)$. (a) Vector distribution of current density in MEA. (b) $x-y$ plane.

\section{Conclusions}

The results obtained by this research are summarized as follows:

(1) The heuristic search for detecting the current distribution, which produces the specified flux distribution, is proposed. It is shown that the detection of the distribution of generation current in MEA is possible by using the proposed heuristic search within acceptable number of iteration.

(2) The detection of the current distribution in MEA is possible from the distribution of static magnetic field around the fuel cell using the proposed 3D heuristic search.

In this paper, the current distribution of only $\mathrm{ON}$ or $\mathrm{OFF}$ regions in MEA is detected, and the divided domains in MEA are $1 \times 10^{-4} \mathrm{~m}^{2}$ (25 elements in MEA). In actual factory, the measurement of the current distribution in 10000 divisions inside MEA is needed. The increase in the division of the current distribution in MEA is a future research subject.

\section{REFERENCES}

1) G. Bender, M. S. Wison and T. Zawodzinski: J. Power Sources 123 (2003) 163-171.

2) A. B. Geiger, R. Eckl, A. Wokaun and G. G. Scherer: J. Electrochem. Soc. 151 (2004) A394-A398.

3) M. Hamalaine, R. Hari, R. J. Ilmoniemi, J. Knuutila and O. V. Lounasmaa: Rev. Modern Phys. 65 (1993) 413-467.

4) R. M. Ward and M. H. Jacobs: J. Mater. Sci. 39 (2004) 7135-7143.

5) Y. Gotoh, N. Takahashi and M. Ozumi: Workshop on Optimization and Inverse Problems in Electromagnetism, (2008) pp. 161-162.

6) N. Takahashi, T. Kitamura, M. Horii and J. Takehara: IEEE Trans. Magn. 36 (2000) 1089-1093.

7) M. Horii, N. Takahashi and T. Narita: IEEE Trans. Magn. 36 (2000) 1085-1088. 